\title{
Utilización de modelos matemáticos para el aprendizaje de aspectos avanzados de Combustión en alumnos de Ingeniería.
}

\author{
Jaime Martin $^{a}$, Antonio García ${ }^{a}$, Joaquín De la Morena ${ }^{a}$ y Javier Monsalve-Serrano ${ }^{a}$

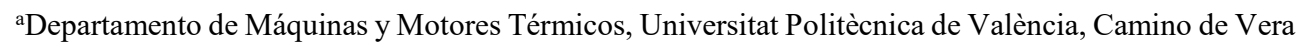 \\ s/n (Edificio 6D), 46022 Valencia (España)
}

\begin{abstract}
The current work sintetizes the main findings regarding the application of mathematical models for advanced combustion physical and chemical aspects to the teaching-learning process in Combustion subjects for Engineering students. These models have been previously developed for research purposes, and then adapted as teaching tools. The aim of this adaptation is to complement the application cases seen in the classroom, where simplified methods are used, with more realistic studies only achievable through computational methods due to their higher mathematical complexity. The paper describes the main characteristics of the tools used, the methodology used to incorporate them to the teaching environment and, finally, evaluates the main impact of their application both in the learning results from the students and their satisfaction about the process.
\end{abstract}

Keywords: combustion, computer-aided learning, termochemistry, diffusion flames.

\begin{abstract}
Resumen
En el presente trabajo se sintetizan los resultados de la aplicación de modelos matemáticos para aspectos físico-químicos avanzados aplicados al proceso de enseñanza-aprendizaje de la asignatura de Combustión para alumnos de lngeniería. Estos modelos han sido desarrollados previamente en el ámbito investigador, y posteriormente adaptados como herramientas docentes. El objetivo de las mismas es complementar los casos vistos en el aula, donde se trabaja con métodos de resolución simplificados, con estudios más realistas que únicamente son abordables computacionalmente debido a la mayor complejidad matemática. En el artículo se presentan los fundamentos de las herramientas utilizadas, se describe la metodología usada para su adaptación al ámbito docente y, finalmente, se evalúa el impacto de su implementación tanto en los resultados de aprendizaje de los alumnos como en su grado de satisfacción final.
\end{abstract}

Palabras clave: combustión, aprendizaje asistido por ordenador, termoquímica, llamas de difusión. 
Utilización de modelos matemáticos para el aprendizaje de aspectos avanzados de Combustión en alumnos de Ingeniería.

\section{Introducción}

En el presente trabajo se ha abordado el potencial del uso de modelos computacionales para la mejora del proceso de enseñanza-aprendizaje en la asignatura de Combustión, actualmente existente en las titulaciones de Grado en Ingeniería Aeroespacial e Ingeniería Mecánica, así como en el Máster en Ingeniería Industrial de la Universitat Politècnica de València.

Una de las dificultades que plantea la enseñanza de dicha asignatura es que, pese a estar en cursos avanzados de los estudios anteriormente descritos, tiene una fuerte carga teórica, por lo que resulta en ocasiones difícil mantener la motivación del alumnado. Por otra parte, el aprendizaje del proceso de combustón resulta generalmente complejo. En primer lugar, la combustión es un fenómeno que incopora elementos de materias muy diversas (química, termodinámica, transmisión de calor, mecánica de fluidos, etc.), por lo que requiere de un esfuerzo de síntesis por parte del alumno. Además, las aplicaciones reales que incorporan procesos de combustión son muy complejas tanto desde un punto de vista químico (Kubota, 2015), por la intervención de múltiples reacciones químicas acopladas, como a nivel físico, por la interacción del flujo turbulento (Turns, 2011). Por este motivo, a nivel docente se suele acudir a modelos teóricos simplificados, que permiten al alumno aprehender los mecanismos principales que actúan sobre el problema, pero que no son del todo representativos de los procesos en aplicaciones reales

Por todos estos motivos se plantean los siguientes objetivos de aprendizaje para la presente la innovación:

- Mejorar el grado de participación e implicación de los alumnos en la asignatura.

- Completar los cálculos teóricos vistos en el aula con ejemplos más cercanos a la aplicación práctica.

- Proporcionar órdenes de magnitud de la influencia de los parámetros más importantes sobre el proceso de combustión.

En este sentido, el empleo de técnicas computacionales se ha presentado, como muy provechoso para el proceso de enseñanza-aprendizaje, especialmente en estudios de Ingeniería (Seyfer y Russell, 1986). Este tipo de herramientas se han desarrollado en el ámbito del Computer Aided Learning (CAL) o aprendizaje asistido por ordenador (Parslow, 1998). Osman y Muir (1994) demostraron que el uso de estas técnicas pueden ayudar a mejorar el rendimiento del proceso de aprendizaje. Por otro lado, Edmonds (1980) también demostró que las habilidades adquiridas con este tipo de técnicas se retienen durante más tiempo. Peruman y Ganesan (2016) concluyeron que el aprendizaje basado en modelos era útil en el caso concreto del estudio de procesos fluido-dinámicos complejos. GutierrezRomero y otros (2017) afirmaron que estas experiencias se perciben por parte de los alumnos como más cercanas a la industria con respecto al trabajo convencional en el aula, por lo que aumenta su nivel de implicación. Finalmente, Burke y otros (2017) mostraron evidencias de que el uso de herramientas computacionales ayudaba a los alumnos a mejorar la comprensión de fenómenos complejos y de sus interacciones, además de ventajas concretas en cuanto al aprendizaje de alumnos extranjeros. 
En resumen, los objetivos básicos del aprendizaje asistido por ordenador son:

- Facilitar la transición desde una clase centrada en el profesor a un proceso de aprendizaje más interactivo.

- Fomentar la participación e implicación del alumnado.

- Asegurar la claridad conceptual y aplicabilidad de los contenidos.

- Crear un entorno de auto-aprendizaje.

Por otra parte, como ya se ha introducido la utilización de modelos complejos permite a los alumnos una aproximación más realista a los procesos de combustión. No obstante, existen multitud de aspectos que podrían considerarse en cuanto al mismo. En primera aproximación, es necesario analizar el problema de la combustión desde un punto de vista energético con el fin de diseñar estos sistemas y mazimizar su rendimiento (Comisión Europea, 2017). Por otro lado, resulta evidente que la combustión tiene además un fuerte impacto sobre el medio ambiente por su contribución a la emisión de gases de efecto invernadero (fundamentalmente dióxido de carbono) como por la producción de otros contaminantes, como los óxidos de nitrógeno o el hollín (Weilenmann y otros, 2005; Kirchstetter y otros, 1999; Archer, 2013).

Para tratar de cubrir estos aspectos se ha decidido abordar la innovación utilizando dos herramientas diferenciadas desarrolladas en dos sesiones en aula informática. En primer lugar, se tratará un modelo general para la estimación de los productos de la combustión bajo la hipótesis de equilibrio químico y su implicación sobre la temperatura adiabática de llama (Law y otros, 2016). Este modelo asume condiciones ideales en los aspectos físicos (mezcla homogénea, presión constante y tiempo de residencia alto), con el objetivo de evaluar únicamente la parte química del fenómeno. Posteriormente, se verá su acoplamiento a un ejemplo concreto de aplicación real, como es el de la combustión de un chorro de combustible líquido (Desantes y otros, 2014). Para ello se han adaptado previamente dos modelos desarrollados internamente en el ámbito investigador del Departamento de Máquinas y Motores Térmicos de la Universitat Politècnica de València para su uso docente. Durante las sesiones se ha propuesto a los alumnos la realización de una serie de estudios paramétricos orientados a mejorar la comprensión de los procesos involucrados en cada problema. En los siguientes apartados se describirá brevemente ambos modelos, la metodología docente utilizada y los resultados obtenidos en cuanto a resultados de aprendizaje y satisfacción de los alumnos. Finalmente, se establecerán las conclusiones globales más importantes del estudio.

\section{Modelos utilizados}

En esta sección se presentarán los fundamentos básicos de los modelos físico-químicos utilizados para la práctica docente, con el fin de comprender mejor los objetivos de aprendizaje cubiertos con cada uno de ellos 
Utilización de modelos matemáticos para el aprendizaje de aspectos avanzados de Combustión en alumnos de Ingeniería.

\subsection{Modelo termoquímico}

El modelo termoquímico usado para el cálculo de las especies existentes en los productos de la combustión, y a partir de los mismos de la temperatura adiabática de llama, se basa en la hipótesis de reacción en equilibrio químico. Según esta, se puede aproximar un proceso real de combustión mediante la siguiente reacción global:

$$
\begin{aligned}
& \mathrm{C}_{n} \mathrm{H}_{\mathrm{m}}+\mathrm{n}_{\mathrm{a}}\left(\mathrm{X}_{\mathrm{N} 2} \mathrm{~N}_{2}+\mathrm{X}_{\mathrm{O} 2} \mathrm{O}_{2}+\mathrm{X}_{\mathrm{H}_{2} \mathrm{O}} \mathrm{H}_{2} \mathrm{O}+\mathrm{X}_{\mathrm{CO} 2} \mathrm{CO} 2+\mathrm{X}_{\mathrm{Ar}} \mathrm{Ar}\right) \rightarrow \mathrm{n}_{1} \mathrm{~N}_{2}+ \\
& \mathrm{n}_{2} \mathrm{O}_{2}+\mathrm{n}_{3} \mathrm{CO}_{2}+\mathrm{n}_{4} \mathrm{H}_{2} \mathrm{O}+\mathrm{n}_{5} \mathrm{CO}+\mathrm{n}_{6} \mathrm{H}_{2}+\mathrm{n}_{7} \mathrm{NO}+\mathrm{n}_{8} \mathrm{OH}+\mathrm{n}_{9} \mathrm{~N}+\mathrm{n}_{10} \mathrm{H}+ \\
& \mathrm{n}_{11} \mathrm{O}+\mathrm{n}_{12} \mathrm{Ar}
\end{aligned}
$$

Donde $n_{i}$ representa el número de moles existente para cada especie de productos, $n_{a}$ el número de moles de aire disponibles por cada mol de combustible y $X_{i}$ las fracciones molares de cada especie que conforma este aire. En las mismas se incluye el $\mathrm{CO}_{2}$ por la posible inclusión de gases de escape recirculados en determinadas aplicaciones. La composición del combustible y del aire, así como la proporción entre ambas (a partir del dosado relativo) son variables de entrada para el usuario. De este modo, quedarían en el modelo doce incógnitas correspondientes a los números de moles de cada uno de los productos $\left(n_{i}\right)$, por los que es preciso introducir doce ecuaciones en el modelo. Cinco de estas ecuaciones vienen de la aplicación directa del balance atómico del carbono, oxígeno, nitrógeno, hidrógeno y argón entre reactivos y productos. Las otras siete proceden en cambio de la aplicación de la hipótesis de equilibrio químico en siete reacciones de disociación asociadas al proceso. Para el cálculo de las reacciones de disociación en equilibrio se hace necesario calcular adicionalmente la temperatura adiabática de llama, por lo que se puede ver que el cálculo de la composición de los productos y de dicha temperatura se encuentran acoplados. Más detalles de este cálculo se pueden obtener en Turns (2011).

\subsection{Modelo de chorro líquido en combustión}

La inyección y combustión de líquidos es habitual en múltiples instalaciones como quemadores de calderas, turbinas de gas o motores de encendido provocado. En el presente modelo se asume que la inyección del combustible se produce sobre una cámara en la que el aire se encuentra en reposo, y que además ésta es suficientemente grande como para que las paredes de la misma no perturben el proceso de mezcla aire-combustible. Además, se asume que el combustible sale de la tobera de inyección con un perfil de velocidad uniforme, y que al producirse la mezcla se forma un chorro con un ángulo de apertura constante y definido como entrada al modelo.

En el modelo se introducen perfiles radiales normalizados tanto de velocidad axial $(u)$ como de fracción de mezcla $(f)$, por lo que se puede reducir el cálculo de todos los parámetros a una aproximación unidimensional. A partir de las condiciones de contorno de gasto másico y flujo de cantidad de movimiento a la salida de la tobera, y teniendo en cuenta una ecuación de estado, es posible resolver la ecuación de conservación de la cantidad de movimiento desde la misma y a lo largo de todo el eje del chorro formado.

Finalmente, en el caso de un chorro reactivo (es decir, en el que se lleva a cabo la reacción de combustión) es necesario cerrar el problema con la ecuación de conservación de la energía.

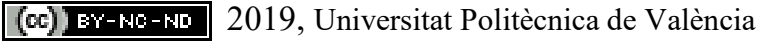


Teniendo en cuenta estas hipótesis y ecuaciones, el proceso de cálculo del programa se puede resumir en el diagrama presentado en la Figura 1. Más detalles sobre el modelo y la metodología de resolución del mismo se pueden encontrar en Desantes y otros (2014).

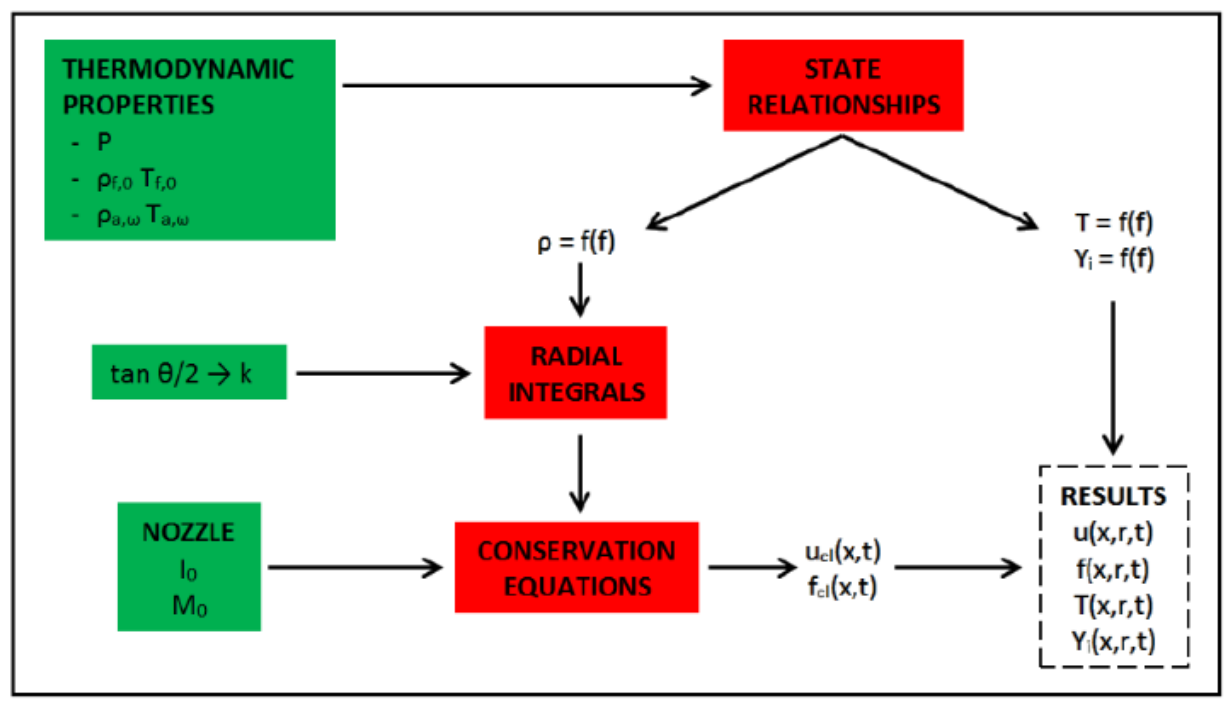

Fig. 1 Esquema de cálculo del modelo de chorro líquido.

\section{Metodología docente}

A continuación, se describen los principales aspectos de la metodología utilizada durante la aplicación docente de los modelos anteriores. Esto incluye la organización de las sesiones en aula informática donde se utilizan los modelos y un resumen de los estudios paramétricos que se plantean a los alumnos.

\subsection{Organización y metodología}

Tal como se ha dicho anteriormente, para el mejor aprovechamiento de las técnicas de aprendizaje asistido por ordenador se plantea el trabajo con ambos modelos en sesiones dedicadas en aula de informática, en las que se trabaja con un número de alumnos reducido. En este sentido, se divide al número total de alumnos (en torno a 200) en grupos de entre 15 y 25 alumnos. La sesión tiene una duración total de 3 horas, estructuradas de la siguiente manera:

a) Introducción teórica (30 minutos). El profesor responsable del grupo introduce los conceptos teóricos más básicos del modelo a utilizar, resaltando las diferencias existentes entre el mismo y la solución más sencilla desarrollada durante las clases de teoría. Además, se ofrecen algunos ejemplos de validación del modelo frente a datos experimentales, obtenidos de trabajos de investigación previos. Esto permite al alumno ganar confianza en la precisión de la herramienta que se dispone a utilizar, incrementando su implicación en la sesión.

b) Tutorial del modelo (15 minutos). De nuevo el profesor explica desde su ordenador la organización del software y el modo de trabajar con el mismo. 
c) Estudios paramétricos guiados (120 minutos). Por parejas, los alumnos deben realizar una serie de estudios paramétricos en los que se varían las principales condiciones de contorno del problema a analizar. Además de realizar los cálculos y reportar los resultados, se pide a los alumnos que respondan a una serie de preguntas, planteadas para guiar el análisis de los mismos.

d) Test de evaluación y encuestas de opinión (15 minutos). Para evaluar el aprovechamiento de la sesión respecto a la comprensión de los conceptos teóricos asociados, se plantea al final de cada sesión un examen de tipo prueba objetiva o test con 5 preguntas y 4 opciones posibles, en las que el alumno debe marcar la opción correcta. Finalmente, se pasa a los alumnos una encuesta con 10 preguntas para establecer su grado de satisfacción con la metodología y materiales utilizados.

\subsection{Estudios paramétricos: modelo termoquímico}

En la sesión dedicada al modelo termoquímico, se plantea un total de 4 estudios paramétricos independientes:

\section{a) Efecto del dosado relativo}

En primer lugar, se plantea variar únicamente el dosado o relación másica combustible-aire utilizado para el cálculo de la reacción química, manteniendo el resto de parámetros constantes. Las condiciones de contorno de este estudio se presentan en la Tabla 1:

Tabla 1. Estudio paramétrico del dosado

\begin{tabular}{|l|l|}
\hline Fuel [-] & Dodecano $\left(\mathrm{C}_{12} \mathrm{H}_{26}\right)$ \\
\hline Fr [-] & $0.5-3(20$ puntos $)$ \\
\hline EGR [\%] & 0 \\
\hline $\mathrm{P}_{\text {ini }}[$ bar] & 1 \\
\hline$T_{R}[\mathrm{~K}]$ & 303 \\
\hline
\end{tabular}

Como se puede apreciar, se mantienen condiciones atmosféricas $\left(1\right.$ bar y $\left.30^{\circ} \mathrm{C}\right)$ con aire estándar (sin recirculación de gases de escape o EGR). El objetivo del estudio será comparar la solución obtenida mediante el cálculo completo con disociación en equilibrio con el cálculo simplificado desarrollado previamente en el aula, basado en una reacción de un solo paso desplazada a productos. Los alumnos podrán apreciar cómo la temperatura alcanzada será más baja como consecuencia de la disociación, y cómo el máximo de la misma no se alcanzará para dosado estrequiométrico sino para un valor ligeramente rico. Así mismo, en cuanto a la composición de los productos, se apreciará cómo al acercarse a valores estequiométricos se produce una desviación significativa en los valores estimados de $\mathrm{CO}_{2} \mathrm{y}$ $\mathrm{H}_{2} \mathrm{O}$, que se acentúa para el caso de dosado rico. 


\section{b) Efecto de la temperatura inicial}

El segundo estudio paramétrico trata de evaluar la influencia de la temperatura inicial de la mezcla aire-combustible. Las condiciones son similares a las de la Tabla 1, pero manteniendo un dosado relativo constante (igual a la unidad) y variando la temperatura inicial entre $300 \mathrm{y}$ $900 \mathrm{~K}$. El objetivo será ver que la temperatura inicial afecta no solo a la temperatura adiabática de llama, que aumentará, sino también a la disociación, por lo que la cantidad obtenida de especies como el $\mathrm{CO}$ y el $\mathrm{H}_{2}$ también aumenta con la temperatura inicial.

\section{c) Efecto de la presión}

De nuevo se parte de los parámetros de la Tabla 1, variando en este caso la presión en un rango de 1 a 100 bar. El objetivo del estudio será que los alumnos vean cómo al aumentar la presión el equilibrio de las reacciones de la disociación se desplaza hacia el sentido que en cada caso minimice el número de moles existente en la mezcla, según el principio de Le Chatêlier. En cualquier caso, se podrá observar cómo el efecto de la presión es el menos significativo de los planteados.

d) Efecto de la composición del aire

Para evaluar la influencia de los resultados respecto a la fracción de oxígeno en el aire se introduce una determinada tasa de recirculación de gases de escape o EGR, definida como el gasto másico de gases recirculados dividido del gasto total entrante a la cámara de combustión. Esta tasa de EGR se varía entre 0 y $50 \%$ en el estudio. Se observará cómo la temperatura adiabática obtenida será significativamente menor como consecuencia de la mayor cantidad de gases inertes disponible en la mezcla inicial, lo que tiene efectos positivos para reducir la cantidad de óxido de nitrógeno (NO) formada.

\subsection{Estudios paramétricos: modelo de chorro líquido}

La Tabla 2 presenta las variaciones paramétricas que los alumnos realizan en el caso del análisis del chorro líquido inerte. En cada condición se resalta en negrita el valor a variar respecto al caso anterior, para simplificar el seguimiento del estudio por parte de los alumnos.

Tabla 2. Estudio paramétrico del chorro inerte

\begin{tabular}{|c|c|c|c|c|c|c|}
\hline Condición & Ángulo $\left[{ }^{\mathbf{}}\right]$ & $\mathbf{d}_{\mathbf{0}}[\mathbf{m m}]$ & $\Delta \mathbf{P}[\mathbf{b a r}]$ & $\boldsymbol{\rho}_{\mathrm{f}}\left[\mathbf{k g} / \mathbf{m}^{3}\right]$ & $\boldsymbol{\rho}_{\mathbf{a}}\left[\mathbf{k g} / \mathbf{m}^{3}\right]$ & $\mathbf{f}_{\text {st }}[-]$ \\
\hline $\mathbf{1}$ & 20 & 0.150 & 800 & 830 & 30 & $1 / 15$ \\
\hline $\mathbf{2}$ & 20 & $\mathbf{0 . 1 2 5}$ & 800 & 830 & 30 & $1 / 15$ \\
\hline $\mathbf{3}$ & 20 & $\mathbf{0 . 1 7 5}$ & 800 & 830 & 30 & $1 / 15$ \\
\hline $\mathbf{4}$ & 20 & 0.150 & 800 & 830 & $\mathbf{2 0}$ & $1 / 15$ \\
\hline $\mathbf{5}$ & 20 & 0.150 & 800 & 830 & $\mathbf{4 0}$ & $1 / 15$ \\
\hline $\mathbf{6}$ & $\mathbf{1 5}$ & 0.150 & 800 & 830 & 30 & $1 / 15$ \\
\hline $\mathbf{7}$ & $\mathbf{2 5}$ & 0.150 & 800 & 830 & 30 & $1 / 15$ \\
\hline
\end{tabular}


Utilización de modelos matemáticos para el aprendizaje de aspectos avanzados de Combustión en alumnos de Ingeniería.

Si se analiza con detalle esta tabla, se puede apreciar cómo la combinación de los casos 1, 2 y 3 permite evaluar el efeto del diámetro de salida de la tobera sobre el comportamiento del chorro. Del mismo modo, combinando los casos 1, 4 y 5 se analiza el efecto de la densidad del aire, y con los casos 1, 6 y 7 el del ángulo de apertura del chorro. Para cada estudio se pide a los alumnos que grafiquen la evolución axial de la velocidad, la fracción de mezcla, el radio estequiométrico y el gasto másico total que atraviesa el chorro. Mediante el análisis combinado de las 4 gráficas, se pide que describan de forma crítica si cada parámetro tiene un efecto positivo o negativo sobre el proceso de mezcla aire-combustible.

Una vez analizado el caso inerte, se pide a los alumnos que realicen un segundo estudio para comprobar el efecto que tiene la reacción de combustión sobre el comportamiento del chorro. Las condiciones de este estudio se detallan en la Tabla 3.

Tabla 3. Estudio paramétrico del chorro inerte

\begin{tabular}{|c|c|c|c|c|c|c|c|}
\hline Condición & Ángulo $\left[^{\mathbf{o}}\right]$ & $\mathbf{d} \mathbf{0}[\mathbf{m m}]$ & $\boldsymbol{\Delta} \mathbf{P}[\mathbf{b a r}]$ & $\boldsymbol{\rho}_{\mathbf{f}}\left[\mathbf{k g} / \mathbf{m}^{\mathbf{3}}\right]$ & $\boldsymbol{\rho}_{\mathbf{a}}\left[\mathbf{k g} / \mathbf{m}^{3}\right]$ & $\mathbf{P}[\mathbf{b a r}]$ & $\mathbf{f L O L [ - ]}$ \\
\hline $\mathbf{8}$ & $20^{\circ}$ & 0.150 & 800 & 830 & 30 & 50 & $\mathbf{0}$ (inerte) \\
\hline $\mathbf{9}$ & $20^{\circ}$ & 0.150 & 800 & 830 & 30 & 50 & $\mathbf{1}$ (reactivo) \\
\hline
\end{tabular}

\section{Resultados}

En esta sección se incluyen los resultados obtenidos por los alumnos respecto a la evaluación de la práctica, así como el análisis de las encuestas de satisfacción realizadas.

\subsection{Tests de evaluación}

La figura 2 muestra los principales resultados obtenidos para la sesión centrada en el análisis termoquímico durante los últimos años en una escala de 0 a 10. Como se puede observar aproximadamente un $70 \%$ de los alumnos obtienen calificaciones iguales o superiores a 8 , lo que confirma la capacidad de la metodología planteada para alcanzar un grado más que satisfactorio de conocimientos por parte de los alumnos. Hay menos de un 15\% del alumnado que no alcanza la calificación mínima esperada de 5. Además, la tendencia es consistente durante los 5 años en los que la metodología ha sido implantada. 


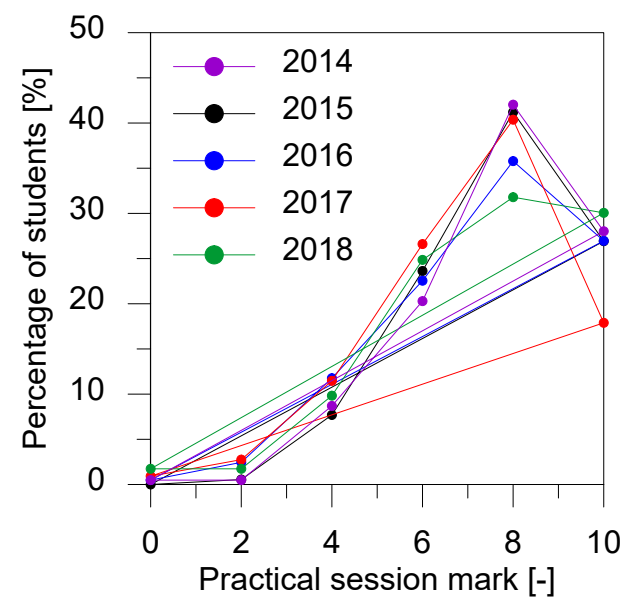

Fig. 2 Resultados de los tests de evaluación del modelo termoquímico.

Algo similar ocurre con los resultados de la sesión centrada en el estudio de chorros líquidos, cuyos resultados se encuentran en la Figura 3. En este caso, se ha optado por representar el valor promedio junto con la dispersión típica en la línea azul, que como se puede apreciar se sitúa siempre por encima de 9 sobre 10.

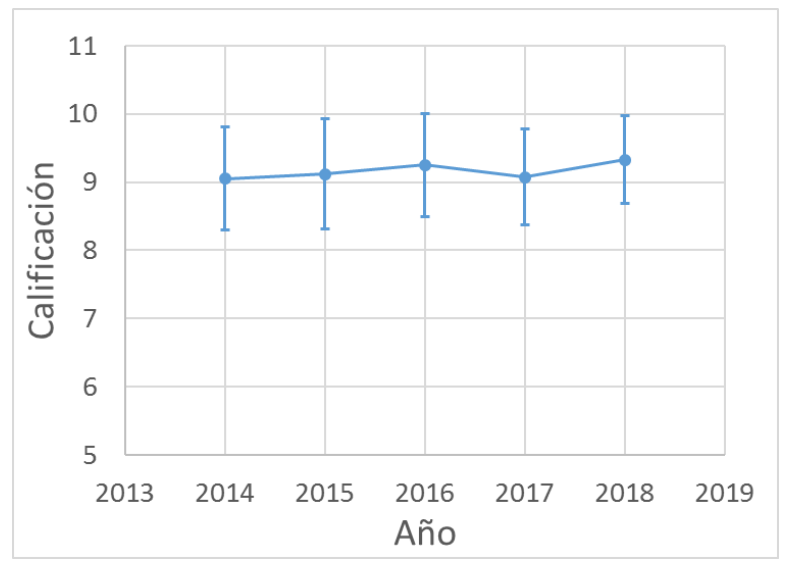

Fig. 3 Resultados de los tests de evaluación del modelo de chorro líquido.

\subsection{Encuestas de satisfacción}

Como se ha descrito anteriormente, la encuesta suministrada a los alumnos se compone de diez preguntas, que indagan sobre diferentes aspectos tanto docentes como organizativos. Estas preguntas se exponen a continuación:

1. Valoración global de la práctica

2. Los contenidos:

2.1. Son interesantes.

2.2. Están relacionados con los contenidos teóricos de la asignatura

2.3. Aplica los conocimientos teóricos de la asignatura 
Utilización de modelos matemáticos para el aprendizaje de aspectos avanzados de Combustión en alumnos de Ingeniería.

3. El profesor:

3.1. Domina la materia

3.2. Explica con claridad

3.3. Ha sido punctual en el inicio y fin de la práctica

4. Recursos:

4.1. El material es adecuado

4.2. Hay suficiente material

4.3. El laboratorio/aula resulta confortable

5. Organización

5.1. La información previa de horarios y lugares es suficiente

5.2. La información de horarios y lugares está disponible a tiempo

De entre todas estas preguntas, se ha decidido analizar únicamente las 4 primeras, que son las enfocadas directamente con la metodología docente aplicada. Esta información se puede apreciar, a nivel promedio (en escala de 0 a 5 ) en la Figura 5 para el grupo correspondiente al Grado en Ingeniería Mecánica, con un tamaño medio de 200 alumnos. Como se puede apreciar, el promedio de las respuestas de todos los alumnos a cada una de las preguntas se encuentra siempre por encima de 4.5, lo que implica que los alumnos se encuentran cómodos y valoran la metodología utilizada.

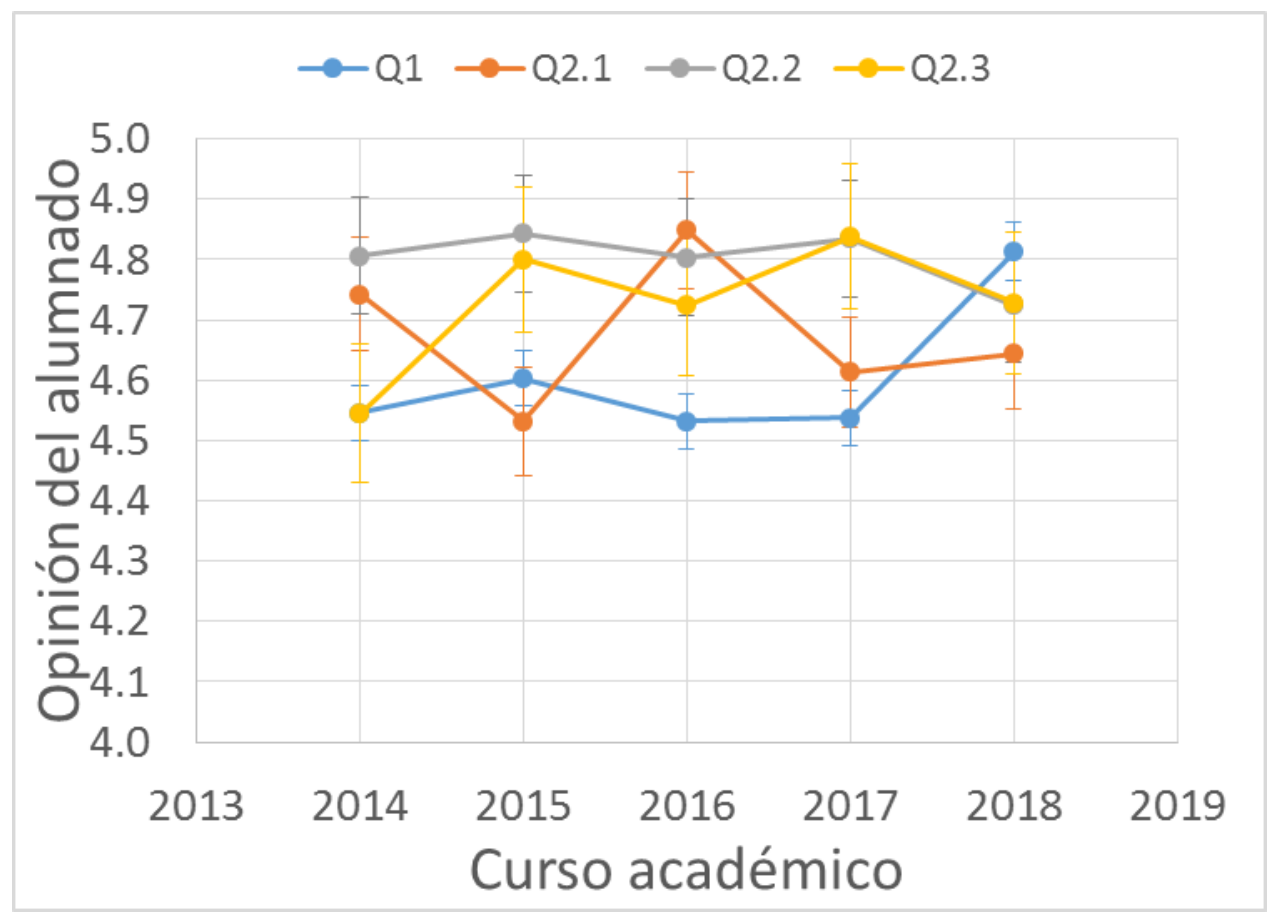

Fig. 4 Resultados de las encuestas de satisfacción de los alumnos 


\section{Conclusiones}

En el presente estudio se ha introducido una metodología para complementar el aprendizaje de los alumnos de Combustión en distintas asignaturas de Grado y Máster de estudios de Ingeniería Aeroespacial, Mecánoica e Industrial mediante el uso de herramientas computacionales. Los modelos utilizados han sido previamente desarrollados y validados en ámbito investigador, y posteriormente adaptados al entorno docente. En concreto se han analizado dos problemas cuya solución precisa no es abordable en el entorno del aula tradicional. El primero está relacionado con el cálculo de la composición y temperatura alcanzadas por los productos de la combustión, generalmente complejo debido al acoplamiento entre ambas magnitudes. El segundo problema es el del desarrollo de un chorro líquido, donde intervienen mecanismos físicos complejos como la atomización, evaporación y mezcla aire-combustible, todos ellos afectados por el nivel de turbulencia del flujo. Cada uno de estos modelos ha sido brevemente descrito en cuanto a sus fundamentos teóricos más significativos.

La metodología docente propuesta se ha basado siempre en la realización de estudios paramétricos con ambos modelos por parte de los alumnos, en sesiones separadas con un número limitado de los mismos. El objetivo principal es que los alumnos adquieran un orden de magnitud de la importancia relativa que cada condición de contorno que afecta al problema tiene sobre su solución. Los estudios están parcialmente guiados mediante una serie de preguntas que ayudan al análisis de los datos obtenidos por parte de los alumnos. El resultado de la metodología se ha medido mediante dos indicadores principales:

- La calificación obtenida en una prueba objetiva tipo test, focalizada sobre los contenidos teóricos desarrollados en la sesión.

- Los resultados de una encuesta de satisfacción de los alumnos.

Ambas métricas han producido resultados muy satisfactorios a lo largo de los cinco años de implantación de la metodología propuesta, lo que confirma la idoneidad de las técnicas de aprendizaje asistido por ordenador en el ámbito ingenieril.

\section{Referencias}

Archer G. Briefing: Particle emissions from petrol cars. Transp Environ 2013:1-4.

Burke RD, De Jonge N, Avola C, Forte B. A virtual engine laboratory for teaching powertrain engineering. Comput Appl Eng Educ 2017;25:948-60. doi:10.1002/cae.21847.

C. Law, A. Makino, T. Lu, On the off-stoichiometric peaking of adiabatic flame temperature. Combustion and Flame, 145 (4), 808-819 (2006).

C. Seyfer, J. Russell, Success story computer managed instruction development. Nonprofit Management Leadership, 25, 5-8 (1986).

Comisión Europea. Energy, transport and environment indicators. Eurostat Pocketbooks, 2017. ISSN:17254566. doi:10.2785/889945.

Desantes JM, Pastor JV, Garcia-Oliver JM, Briceño FJ. An experimental analysis on the evolution of the transient tip penetration in reacting Diesel sprays. Combust Flame 2014;161:2137-50. doi:10.1016/j.combustflame.2014.01.022. 
Utilización de modelos matemáticos para el aprendizaje de aspectos avanzados de Combustión en alumnos de Ingeniería.

E. Edmonds, Where Next in Computer Aided Learning?, British Journal of Educational Technology, 11, 97104 (1980).

G. Parslow, Computer aided learning, Biochem. Educ., 25, 96-96, (1997).

Gutiérrez-Romero JE, Zamora-Parra B, Esteve-Pérez JA. Acquisition of offshore engineering design skills on naval architecture master courses through potential flow CFD tools. Comput Appl Eng Educ 2017;25:48-61. doi:10.1002/cae.21778.

Kirchstetter TW, Harley RA, Kreisberg NM, Stolzenburg MR, Hering S V. On-road measurement of fine particle and nitrogen oxide emissions from light- and heavy-duty motor vehicles. Atmos Environ 1999;33:2955-68. doi:10.1016/S1352-2310(99)00089-8.

L. Osman, A. Muir, Computer skills and attitudes to computer-aided learning among medical students. Medical Education, 28, 381-385 (1994).

N. Kubota, Thermochemistry of Combustion. Propellants and Explosives, N. Kubota (Ed.), 2015.

Perumal K, Ganesan R. CFD modeling for the estimation of pressure loss coefficients of pipe fittings: An undergraduate project. Comput Appl Eng Educ 2016;24:180-5. doi:10.1002/cae.21695.

Turns, S.R. An introduction to Combustion :Concepts and Applications. Mc. Graw-Hill Education (Nueva York, EEUU), 2011. ISBN-13: 978-0073380193

Weilenmann M, Soltic P, Saxer C, Forss AM, Heeb N. Regulated and nonregulated diesel and gasoline cold start emissions at different temperatures. Atmos Environ 2005;39:2433-41. 